\title{
miR-142-3p expression correlates with operational tolerance
}

In a recent study to determine mechanisms by which operational tolerance is achieved, researchers compared the microRNA expression profiles in peripheral blood mononuclear cells from operationally tolerant patients with those from stable kidney transplant recipients treated with conventional immunosuppression. Minimizing immunosuppression or even achievement of drug-free tolerance is a major goal in organ transplantation. "Our research focuses on understanding tolerance mechanisms and looking for biomarkers that could predict or diagnose tolerant patients among stable transplanted patients on standard immunosuppression and who could be weaned off safely," says investigator Sophie Brouard.

MicroRNA profiling of peripheral blood mononuclear cells from kidney transplant patients identified eight differentially expressed microRNAs, of which miR142-3p was the highest expressed. The expression of miR-142-3p in T cells did not differ between operationally tolerant patients and stable transplant patients, whereas expression of miR-142-3p was significantly higher in B cells from operationally tolerant patients. The expression of miR-142-3p was not affected by the absence of immunosuppressive drugs or time post-transplantation.

\section{$4 . . . e x p r e s s i o n$ of miR-142-3p was significantly higher in B cells from operationally tolerant patients 77}

Using gene microarrays, Danger et al. showed that overexpression of miR-142-3p in the Raji B-cell line modulated almost 1,000 genes, many of which related to immune response, B-cell activation and cell communication. Transfection of miR-142-3p led to the upregulation of key molecules that have been previously identified in operationally tolerant patients, including MS4A1 (CD20), CD38 and BANK1. By contrast, TGFBR1 was downregulated. Stimulation of B cells with TGF- $\beta$ increased miR-142-3p expression. The expression of TGFB is increased in B cells from operationally tolerant patients in the absence of a concomitant increase in TGFBR1 expression, which suggests that a negative feedback loop between miR$142-3 p$ and TGF- $\beta$ might contribute to the maintenance of tolerance.

"We are investigating the function of miR-142-3p and TGF- $\beta$ feedback in B lymphocytes," says Richard Danger from the study group. "In the future, we will also aim to identify prognostic biomarkers of operational tolerance in order to improve patient outcome and quality of life, free from the adverse effects of immunosuppression."

Helene Myrvang

Original article Danger, R. et al. Upregulation of miR$142-3 p$ in peripheral blood mononuclear cells of operationally tolerant patients with a renal transplant. J.Am. Soc. Nephrol. doi:10.1681/ASN.2011060543 